\title{
Incidence and classification of bifid mandibular canals using cone beam computed tomography
}

\section{George Borja de Freitas ${ }^{1}$, Alessandra de Freitas e Silva ${ }^{1}$, Lucas Alexandre Morais ${ }^{2}$, Milena Bortolotto Felippe Silva ${ }^{1}$, Thiago Coelho Gomes da Silva ${ }^{2}$, Luiz Roberto Coutinho Manhães Júnior ${ }^{1}$}

\author{
${ }^{1}$ São Leopoldo Mandic - SLMandic, Dental School, Department of Oral and Maxillofacial Radiology, São Paulo, SP, Brazil \\ ${ }^{2}$ Universidade de Pernambuco - UPE, Pernambuco Dental School, Department of Oral and Maxillofacial Surgery, Recife, PE, Brazil
}

\begin{abstract}
Aim: To determine the prevalence and classification of bifid mandibular canals using cone beam computed tomography (CBCT). Methods: The sample comprised 300 CBCT scans obtained from the Radiology and Imaging Department database at São Leopoldo Mandic Dental School, Campinas, SP, Brazil. All images were performed on Classic I-Cat $₫$ CBCT scanner, with standardized voxel at $0.25 \mathrm{~mm}$ and $13 \mathrm{~cm}$ FOV (field of view). From an axial slice $(0.25 \mathrm{~mm})$ a guiding plane was drawn along the alveolar ridge in order to obtain a cross-section. Results: Among 300 patients, 188 (62.7\%) were female and 112 (37.3\%) were male, aged between 13 to 87 years. Changes in the mandibular canal were observed in 90 patients, $30.0 \%$ of the sample, 51 women (56.7\%) and 39 men (43.3\%). Regarding affected sides, $32.2 \%$ were on the right and $24.5 \%$ on the left, with $43.3 \%$ bilateral cases. Conclusions: According to the results obtained in this study, a prevalence of $30 \%$ of bifid mandibular canals was found, with the most prevalent types classified as B (mesial direction) and bilateral.
\end{abstract}

Keywords: anatomy; mandible; classification.

\section{Introduction}

Surgical procedures such as exodontia of mandibular third molars, osteotomy, mandibular anesthetic technique, bone remodeling and implant placement involve the risk of injuring the mandibular alveolar nerve, buccal nerve and lingual nerve, with consequent transient or permanent paresthesia. The mandibular nerve has a complex pathway, originating from the trigeminal ganglion and branching down to the inferior alveolar nerve in the infratemporal fossa. The mandibular canal is located within the ramus and body of the mandible, creating a pathway that begins at the mandibular foramen, externalizing in the mental foramen. This pathway may or may not follow an intraosseous route towards the mental region as a single canal. It is also characterized by curvatures along a posterior anterior direction, crossing obliquely the entire body of the mandible. Topographically, it is located

Received for publication: November 09, 2015 Accepted: December 13, 2015

Correspondence to: George Borja de Freitas Rua Dr. José Rocha Junqueira, 13 Bairro Ponte Preta

CEP:13045-755 Campinas - SP Phone: +55 1935183600 +55 87996570044 E-mail: george_borja@hotmail.com closer to the inner bone wall up to the mesial aspect of the first molar, moving across to the outer bone wall until it reaches the mental foramen'.

The radiographic appearance of the mandibular canal is characterized by a radiolucent strip between two radiopaque lines ${ }^{2}$ generally as a single structure, presenting different positions inside the body of the mandible in both the upperlower and the medial-lateral directions ${ }^{3}$ with occasional duplicate pathways and bifurcations ${ }^{4}$, and even trifurcations ${ }^{5}$ in some cases.

Knowledge of the jaw anatomy and the path of the mandibular alveolar 
nerve in the mandibular canal is of great importance to dentists, especially for those planning orthognathic surgery, mandibular reconstruction, extraction of third molars or installation of osseointegrated implants ${ }^{6-7}$.

It is therefore paramount to investigate the frequency and classification of bifid mandibular canals using CBCT to aid surgical planning at posterior regions of the mandible, minimizing the risk of accidents and operative complications.

\section{Material and methods}

This study was approved by the Research Ethics Committee of the São Leopoldo Mandic Dental School, Campinas, SP, Brazil (Process number 811.741) in agreement with Resolution No. 466/12 by the Brazilian National Health Council. The sample was established retrospectively within a three-year interval, using the Department of Radiology image database at São Leopoldo Mandic Dental School, Campinas, SP, Brazil. CBCT images from 500 patients who had undergone CBCT imaging for diagnostic or treatment planning purposes were examined, from which 300 images were selected according to the inclusion and exclusion criteria described below.

The sample comprised CBCT scans from both male and female patients aged between 13 to 87 years. Image selection was performed at random, i.e., regardless of ethnicity, gender, age or presence/absence of teeth. Only images with satisfactory tomographic quality were considered. Images were excluded if the patient had a history of trauma and bone lesions in the mandible as well as orthognathic surgery or restorative bone procedures in the posterior mandible.

Images were selected using the Classic I-Cat ${ }^{\circledR}$ (Imaging Sciences International, Hatfield, PA, USA) with standardized voxel at $0.25 \mathrm{~mm}$, FOV (Field of view) of $13 \mathrm{~cm}$ and acquisition time of $40 \mathrm{~s}$ according to the manufacturer's standards and useful radiation time of $6.6 \mathrm{~s}$. The settings used for the acquisitions were those pre-established by default, i.e. $120 \mathrm{kV}$ fixed and 5 to $7 \mathrm{~mA}$ variation, according to the used resolution.

All images were processed and adjusted using XoranCat ${ }^{\circledR}$ software (Xoran Technologies, Ann Arbor, MI. USA). CT image analysis was performed on the tomography workstation, correcting the anatomical planes using the multiplane reconstruction page (MPR).

Only bifid canals with a diameter larger than $1 \mathrm{~mm}$ were included in this study, aiming to achieve clear clinical relevance and standardization of the results. Images were selected by chronological order in which they were taken on XoranCAT scanner software to highlight those with alterations in the mandibular canal (Figure 1).

All images were evaluated in a quiet environment with dim lighting by a single operator, a specialist in Oral Radiology with experience in CBCT scans. The analysis was performed on cross sections of three planes (axial, sagittal and coronal), always following the path of the mandibular canal.

All analyses and evaluations were performed using the Xoran 3.0.34 program (Xoran) in multiplanar reconstructions of three slicing planes: axial, coronal and sagittal. A $3 \times 3$ filter was used to sharpen the $0.25-\mathrm{mm}$ thick images.

Identification of the mandibular canal was optimized by applying small changes to the slicing plane along the bone ridge, as well as corrections in brightness, contrast and image filter, since the mandibular canal is not linear and must be individualized for each side. Whenever a bifid canal was detected, oblique slices were applied to obtain images in the buccolingual direction.

The frequency of bifid mandibular canals was evaluated in the scans against gender, age and affected side and described in terms of percentage and subsequent descriptive analysis.
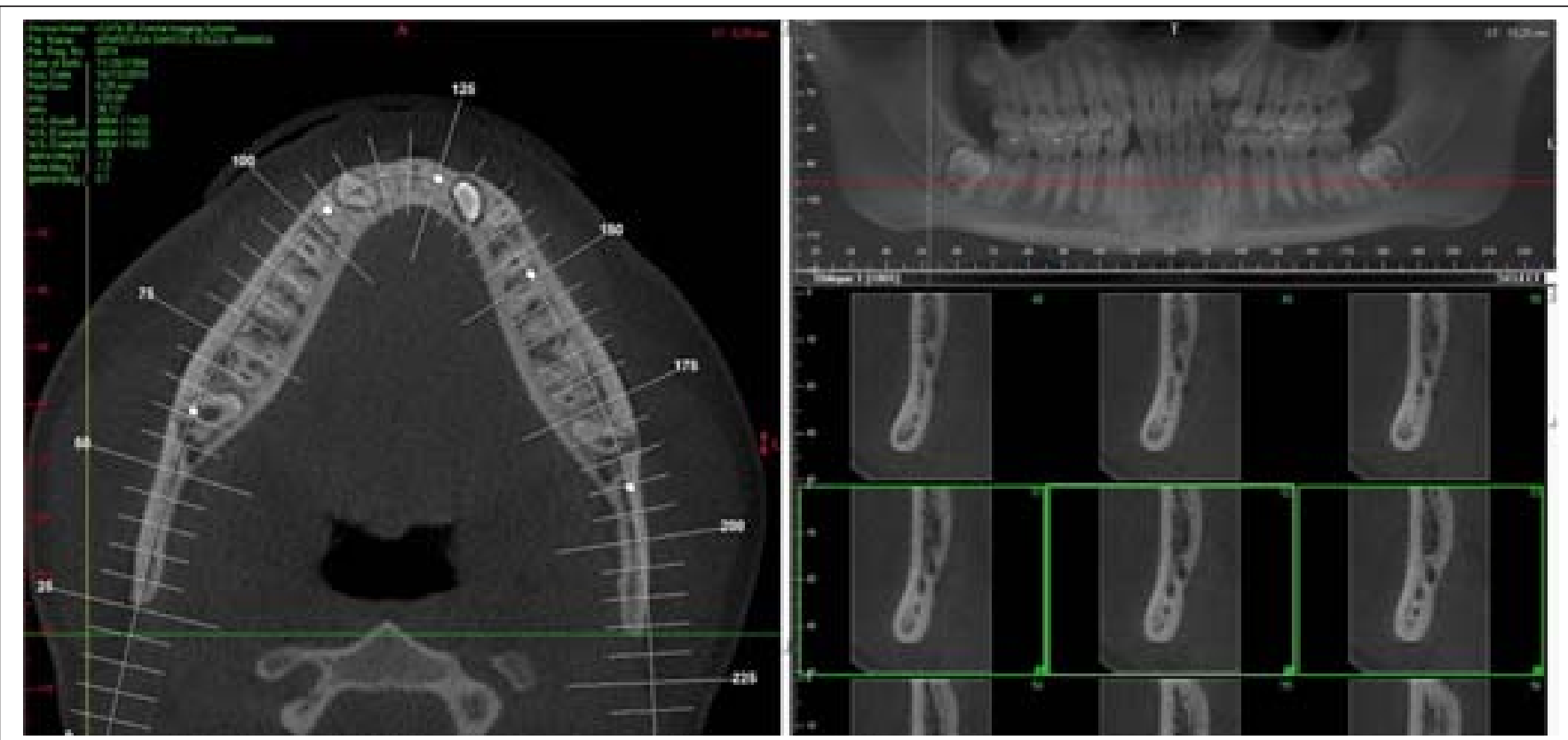

Fig. 1 - Illustration of the methods used to evaluate the CT images. A - Axial reconstruction tracing the jaw line to obtain the cross-sections; B - Panoramic reconstruction C - Cross-sectional reconstruction. 
An Excel spreadsheet (Microsoft, Seattle, WA, USA) was used for data collection to identify the image number, presence of bifid mandibular canals, type of bifid canal, affected side, age and gender. Mandibular canal bifurcations were classified into six distinct categories: Classes A, B, C, $\mathrm{D}, \mathrm{E}$ and $\mathrm{F}$, according to the classification by Naitoh et $\mathrm{al} .^{2}$ (Table 1), which is based on the pathway of the bifid canal, starting from the mandibular foramen. View of a tomographic and clinical changes of the mandibular canal are illustrated in Figures 2 and 3, respectively.

The statistical analysis of CBCT images from 300 participants was descriptive of gender and age in both absolute numbers (n) and percentages (\%). The Student $t$ test for independent samples was applied to check for age differences between genders.

The findings relating to bifid mandibular canals on CBCT were described as absolute and relative frequencies, according to gender and location (right unilateral, left unilateral and bilateral). Additionally, associations between bifid mandibular canals, gender and location were investigated using Fisher's exact and chi-square tests.

The classification of bifid mandibular canals was also analyzed descriptively by frequencies.

Statistical calculations were performed on SPSS 20 (SPSS INC., Chicago, IL, USA) and BioEstat 5.0 (Fundação Mamirauá, Belém, PA, Brazil) at a significance level of 5\% (0.05).

Table 1 - Classification of bifid mandibular canals:

\begin{tabular}{|c|c|}
\hline Class & Direction \\
\hline ass $A$ & $\begin{array}{l}\text { Buccal direction - bifid mandibular canal towards the buccal surface } \\
\text { of the mandible }\end{array}$ \\
\hline Class B & $\begin{array}{l}\text { Mesial direction - bifid mandibular canal towards the mesial or anterior } \\
\text { aspect of the mandible }\end{array}$ \\
\hline Class C & Alveolar direction - bifid mandibular canal towards the alveolar ridge \\
\hline Class D & Retromolar direction - bifid mandibular canal towards retromolar aspect \\
\hline Class E & $\begin{array}{l}\text { Lingual direction - bifid mandibular canal towards the lingual surface of } \\
\text { the mandible }\end{array}$ \\
\hline ass $\mathrm{F}$ & $\begin{array}{l}\text { Base of the mandible - bifid mandibular canal towards the lower } \\
\text { aspect of the mandible or the base of the mandible }\end{array}$ \\
\hline
\end{tabular}

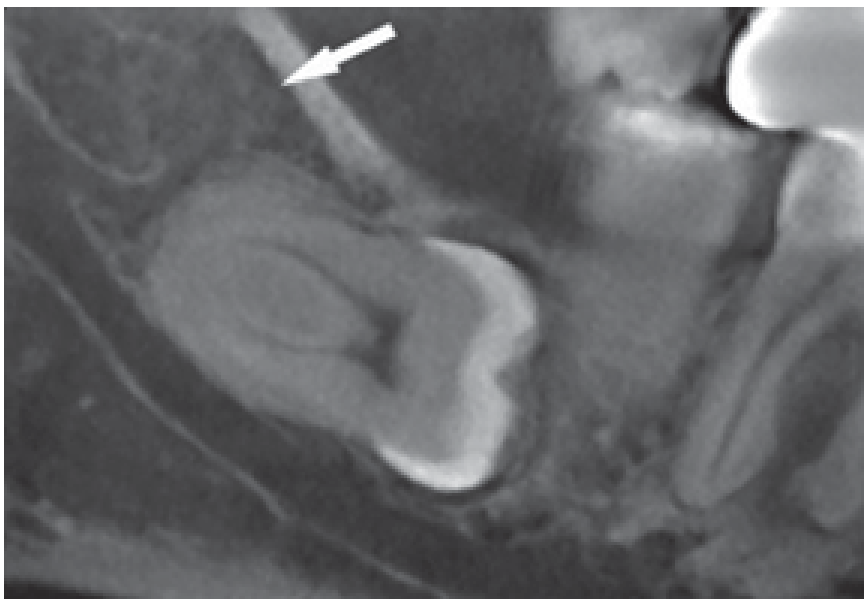

Fig. 2 - CT image showing a retromolar accessory canal (Class D) in close proximity with the unerupted third molar.

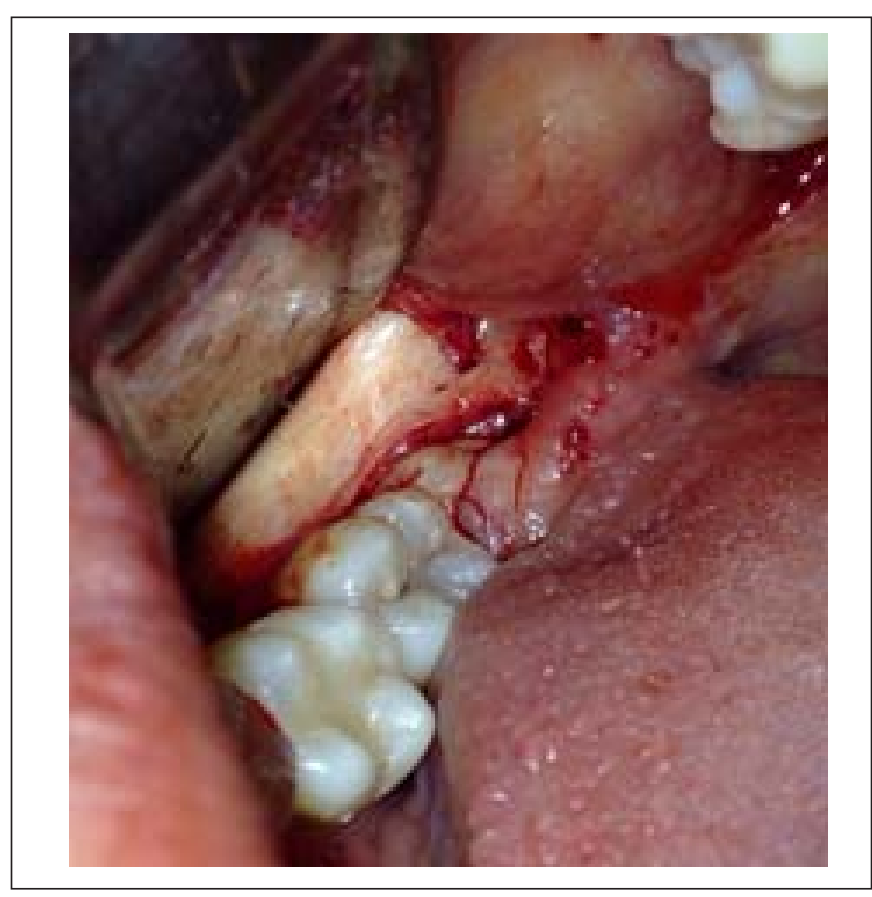

Fig. 3 - Clinical photograph showing a retromolar accessory canal (Class D) in close proximity with the unerupted third molar.

\section{Results}

Descriptive data revealed that $112(37.3 \%)$ images were from males whilst $188(62.7 \%)$ were from females and the 300 patients were aged between 13 and 87 years with a mean age of 48.4 years ( $\mathrm{SD} \pm 15.0$ years). Male individuals were aged between 13 and 77 years (mean 46.4 years, SD \pm 16.1 years), whereas the females were aged between 14 and 87 years (mean was 49.5 years, $S D \pm 14.2$ years). The Student $t$ test for independent samples revealed no difference in age between males and females in this sample $(p=0.077)$.

Regarding the number of canals, $210(70.0 \%)$ images revealed a single mandibular canal, whereas 90 images revealed the presence of bifid mandibular canals, indicating that the prevalence of this event in the study sample was $30.0 \%$. Among the females, $27.1 \%$ (51 out of 188 participants) had bifid mandibular canals against $34.8 \%$ in males (39 of 112). The Fisher's exact test revealed no significant difference between genders (Table 2).

Table 3 shows the absolute and relative frequencies of mandibular canals according to location, indicating that 29 of 90 cases $(32.2 \%)$ of bifid mandibular canals occurred exclusively on the right side, while 22 cases $(24,5 \%)$ were on the left side. Thirty-nine participants $(43.3 \%)$ had bilateral bifid mandibular canals. The chi-square test revealed that the proportion of patients with unilateral bifid canals located either on the right or on the left sides were significantly lower than the proportion of subjects with bilateral bifid mandibular canals $(\mathrm{p}=0.026)$.

Among the $39 \mathrm{CBCT}$ from male patients with bifid mandibular canals, $41.0 \%$ were located exclusively on the right side, $23.1 \%$ on the left and only $35.9 \%$ were bilateral. 
Table 2 - Absolute (n) and relative (\%) frequencies of bifid mandibular canals in CBCT images according to gender

\begin{tabular}{lllll}
\hline Gender & \multicolumn{2}{c}{ Bifid Mandibular Canals } & Total & p-value* \\
& Present & Absent & & \\
Male & $39(34.8 \%)$ & $73(65.2 \%)$ & $112(37.3 \%)$ & \\
Female & $51(27.1 \%)$ & $137(72.9 \%)$ & $188(62.7 \%)$ & 0.193 \\
\hline Total & $90(30.0 \%)$ & $210(70.0 \%)$ & $300(100.0 \%)$ & \\
\hline
\end{tabular}

* the p-value refers to the Fisher's exact test.

Table 3 - Absolute (n) and relative (\%) frequencies of bifid mandibular canals in CBCT images according to location

\begin{tabular}{|c|c|c|c|}
\hline \multirow[t]{2}{*}{ Location* } & \multicolumn{2}{|c|}{ Bifid Mandibular Canals } & \multirow[t]{2}{*}{$p$-value** } \\
\hline & Present & Absent & \\
\hline Unilateral left & $29(32.2 \%)$ & $61(67.8 \%)$ & \\
\hline Unilateral right & $22(24.5 \%)$ & $68(75.5 \%)$ & 0.026 \\
\hline Bilateral & $39(43.3 \%)$ & $51(56.7 \%)$ & \\
\hline \multirow[t]{2}{*}{ Location male } & \multicolumn{2}{|c|}{ Bifid Mandibular Canals } & $p$-value \\
\hline & Present & Absent & \\
\hline Unilateral left & $16(41.0 \%)$ & $23(59.0 \%)$ & \\
\hline Unilateral right & $9(23.1 \%)$ & $30(76.9 \%)$ & 0.223 \\
\hline Bilateral & $14(35.9 \%)$ & $25(64.1 \%)$ & \\
\hline \multirow[t]{2}{*}{ Location female } & \multicolumn{2}{|c|}{ Bifid Mandibular Canals } & $p$-value \\
\hline & Present & Absent & \\
\hline Unilateral left & $13(25.5 \%)$ & $38(74.5 \%)$ & \\
\hline Unilateral right & $13(25.5 \%)$ & $38(74.5 \%)$ & 0.015 \\
\hline Bilateral & $25(49.0 \%)$ & $26(51.0 \%)$ & \\
\hline
\end{tabular}

The chi-square test revealed no difference between the proportions of bifid mandibular canals in different locations (left, right and bilateral, $\mathrm{p}=0.223$ ) (Table 3).

In the 90 patients with bifid mandibular canals, 39 males and 51 females, there were 129 accessory canals. As shown in Figure 4, 86 of them $(66.7 \%)$ were mesially oriented (Class B), against $25(19.4 \%)$ in the retromolar direction (Class D). The E (lingual) and F (base of the mandible) categories were observed in $6(4.6 \%)$ and $12(9.3 \%)$ cases, respectively. No cases were found of bifid mandibular canals classified as Class A (buccal direction) or C (alveolar direction), as illustrated in Figure 4.

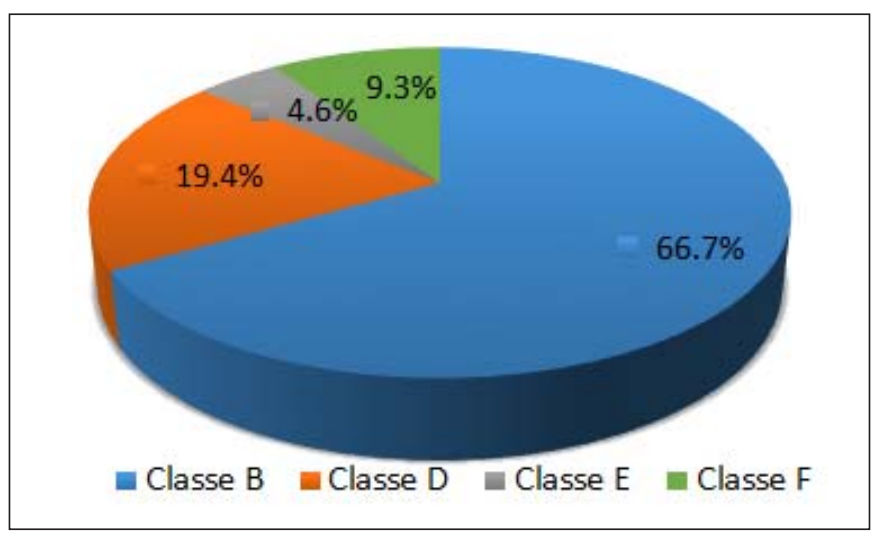

Fig. 4 - Pie chart of the relative frequency (\%) of bifid mandibular canals in cone beam computed tomography, according to pathway classification. Class B: mesial or anterior direction; Class D: retromolar direction; Class E: lingual direction; Class F: base of the mandible direction.

\section{Discussion}

The mandibular canal is most often found as a single one, but canal duplication may occur, and they are also known as bifid canals, which are among the most reported anatomical variations of the mandibular canal in the literature. They can be grouped into different classifications, as proposed by various authors ${ }^{8-9}$. Chávez-Lomeli et al. ${ }^{10}$ questioned the definition of the term "bifid" assigned to the mandibular canal with variation in number, claiming there is no such division of the mandibular canal into two or more canals, but the persistence or non-fusion of embryologically defined branches.

Neves et al. ${ }^{11}$ reported that the mandibular canal has been extensively investigated with regards to its location and pathway, as well as the possible variations in its normal anatomy, which varies considerably, displaying accessory canals with different configurations. Naitoh et al. ${ }^{2}$ reported that these accessory canals may occasionally be observed in panoramic radiographs and may contain a neurovascular bundle.

According to Fukami et al. ${ }^{12}$, the vast majority of dentists have no knowledge of the anatomical variations of the mandibular canal and these variations may have a number of clinical implications if not identified prior to invasive interventions. According to Neves et al. ${ }^{11}$ it is up to the dentist to recognize the possible anatomical variations of the mandibular canal in order to reduce the risk of failure during surgical or anesthetic approaches. For Orhan et al. ${ }^{13}$ knowledge of the anatomy of this canal is essential for successful dental interventions and regional mandibular anesthesia, as well as dental implant placement and jaw surgery.

For Kuribayashi et al. ${ }^{14}$ anatomical variations of the mandibular canal can be identified on panoramic radiographs. However, when more precise information on the path of this canal is required, the most appropriate imaging techniques are the CT scans. Naitoh et $a .^{2}$ compared panoramic radiographs with $\mathrm{CBCT}$ and were able to visualize bifid mandibular canals and accessory foramens in $48.6 \%$ of cases where they were not noted on the panoramic radiographs.

In the present study, bifid mandibular canals were observed in $30.0 \%$ of cases. Previous studies with panoramic radiographs reported incidences of less than $1 \%{ }^{6,9}$. Studies with CBCT images have shown a much higher incidence, with prevalence ranging from $15.6 \%$ to $65 \%{ }^{2,13-15}$, thus reiterating that conventional radiographs are not suitable to detect anatomical variations of the mandibular canal. The differences in incidence may be related to ethnic, geographical as well as methodological differences.

According to Ohran et al. ${ }^{13}$ there are several methods to 
classify anatomical alterations of the mandibular canal, which take into account the characteristics of bifid canals, associations with additional foramens, width and length. Naitoh et al. ${ }^{2}$ suggested the classification of bifid mandibular canals into four types: buccolingual (type I), mesial direction (type II), alveolar ridge direction (type III) and retromolar direction (type IV). In our study, a more comprehensive classification was used, taking into account directions not included in previous classifications, Class A (buccal direction), Class B (mesial direction), Class C (alveolar direction), Class D (retromolar direction), Class E (lingual direction) and Class $\mathrm{F}$ (mandible base direction).

In this study, we found a $19.4 \%$ prevalence of retromolar canals and $7.33 \%$ of additional foramens. Sawyer \& Kiely apud $^{16}$ found a prevalence of retromolar foramens in the order of $7.7 \%$ of cases with significant occurrence of accessory mandibular foramens. Bilecenoglu \& Tuncer ${ }^{16}$ reported a prevalence of $25 \%$ for the retromolar foramen and demonstrated histologically that these canals contained myelin fibers, an artery and numerous venules, which provided innervation to part of the third molar and also the mucosa of the retromolar area.

Regarding the affected side, the right side was most affected in the present study, $32.2 \%$ against $24.5 \%$ on the left side. These findings are corroborated by Ohran et al. ${ }^{13}$ and Carvalho ${ }^{17}$, who also reported a higher prevalence on the right side of about 57.2 to $62.5 \%$.

Some auhors ${ }^{6,9,13,17}$ reported a higher prevalence of bifid mandibular canals among women. No significant difference in the prevalence of bifid mandibular canals was however observed between genders in the used dataset. In the present study, the mean age of the patients affected by changes in the mandibular canal was 48.4 years. This was consistent with the study by Running et al. ${ }^{18}$ who reported a mean age of 48.2 years.

Other studies also reported the use of CBCT to investigate the prevalence of bifid mandibular canals ${ }^{13-14,17}$. In the present study, as well as in the cited literature, CBCT is regarded as appropriate for assessing anatomical variations of the mandibular canal, in view of the difficulties when using conventional radiographs.

According to the results obtained in this study, a prevalence of $30 \%$ of bifid mandibular canals was reported, with the most prevalent types being class B (mesial direction) and bilateral.

\section{References}

1. Kawai T, Asaumi R, Kumazawa Y, Sato I, Yosue T. Observation of the temporal crest canal in the mandibular ramus by cone beam computed tomography and macroscopic study. Int J CARS. 2014 9: 295-299.

2. Naitoh M, Hiraiwa $Y$, Aimiya $H$, Gotoh K, Ariji E. Observation of bifid mandibular canal using cone beam computed tomography. Int J Oral Maxillofac Implants. 2009, 24: 155-9.

3. Neves SN, Nascimento CC, Oliveira ML, Almeida SM, Bóscolo FN. Comparative analysis of mandibular anatomical variations between panoramic radiography and cone beam computed tomography. Oral Maxillofac Surg. 2014; 18: 419-24.
4. Niek L, Gerlach MD, Gert J, Thomas JJ, Frits A. Reproducibility of 3 different tracing methods based on cone beam computed tomography in determining the anatomical position of the mandibular canal. J Oral Maxillofac Surg. 2010; 68: 811.

5. Oliveira-Santos C, Souza PH, De Azambuja Berti-Couto S, Stinkens L, Moyaert K, Van Assche N, et al. Characterization of additional mental foramina through cone beam computed tomography. J Oral Rehabil. 2011; 38: $595-600$.

6. Imada TS, Fernandes LM, Centurion BS, de Oliveira-Santos C, Honório HM, Rubira-Bullen IR. Accessory mental foramina: prevalence, position and diameter assessed by cone-beam computed tomography and digita panoramic radiographs. Clin Oral Implants Res. 2014; 25: e94-e99.

7. Mizbah K, Gerlach N, Maal TJ, Bergé SJ, MeijerGJ. Bifid and trifid mandibular canal. A coincidental finding. Ned Tijdschr Tandheelkd. 2010; 117:616-8.

8. Langlais RP, Broadus R, Glass BJ. Bifid mandibular canals in panoramic radiographs. JAm DentAssoc. 1985; 110: 923-6.

9. Nortjé C J, Farman AG, Joubert J JV. The radiographic appearance of the inferior dental canal: an additional variation. Br J Oral Surg. 1977; 15: 171-2.

10. Chávez-Lomeli ME, Mansilla-Lory J, Pompa JA, Kjaer I. The human mandibular canal arises from three separate canals innervating different tooth groups. J Dent Res. 1996; 75: 1540-4.

11. Neves FS, Almeida SM, Bóscolo FN, Haiter-Neto F, Alves MC, Rebello IC, et al. Risk assessment of inferior alveolar neurovascular bundle by multidetector computed tomography in extractions of third molars. Surg Radiol Anat. 2012; 34: 619-24.

12. Fukami K, Shiozaki K, Mishima A, Kuribayashi A, Hamada Y, Kobayashi K. Bifid mandibular canal: confirmation of limited cone beam CT findings by gross anatomical and histological investigations. Dentomaxillofac Radiol. 2012; 41: 460-5.

13. Orhan K, Aksoy S, Bicenoglu B, Sakul BU, Paksoy CS. Evaluation of bifid mandibular canals with cone beam computed tomography in a Turkish adult population: a retrospective study. Surg Radiol Anat. 2011; 33: 501-7.

14. Kuribayashi A, Watanabe H, Imaizumi A, Tantanapornkul W, Katakami K, Kurabayashi T. Bifid mandibular canals: cone beam computed tomography evaluation. Dentomaxillofac Radiol. 2010; 39: 235-9.

15. Oliveira-Santos C, Souza P H C, Berti-Couto S A, Stinkens L, Moyaert $\mathrm{K}$, Rubira-Bullen IRF, et al. Assessment of variations of the mandibular canal through cone beam computed tomography. Clin Oral Invest. 2012; 16: 387-93. Doi: 10.1007/s00784-011-0544-9.

16. Bilecenoglu B, Tuncer N. Clinical and anatomical study of retromolar foramen and canal. J Oral Maxillofac Surg. 2006; 64: 1493-7.

17. Mizbah K, Gerlak N, Maal TJ, Bergé SJ, Meijer GJ. The clinical relevance of bifid and trifid mandibulars canals. Oral Maxillofac Surg. 2012; 16: 147-51.

18. Correr GM, Iwanko D, Leonardi DP, Ulbrich LM, Araújo MR, Deliberador TM. Classification of bifid mandibular canals using cone beam computed tomography. Braz Oral Res. 2013; 27: 510-6. 